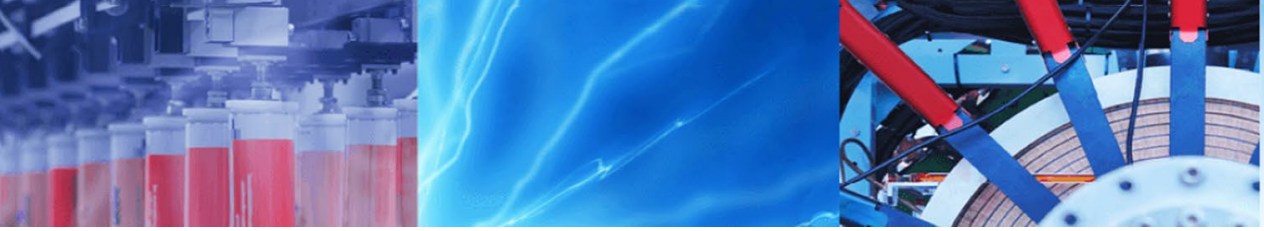

Research Article

\title{
Synthesis of functionalized magnetite nanoparticles using only oleic acid and iron (III) acetylacetonate
}

\author{
Eupídio Scopel ${ }^{1,3} \cdot$ Patrick Pires Conti $^{1,4} \cdot$ Daniel Grando Stroppa $^{2} \cdot$ Cleocir José Dalmaschio $^{1}$ (i)
}

(c) Springer Nature Switzerland AG 2019

\begin{abstract}
The solvothermal method is a way to obtain magnetite nanoparticles with uniformity in shape and size, good dispersibility and functionalized surface. These properties are desirable in many applications in materials science and medical area. Although methods for obtaining nanoparticles with these properties are reported, the most their use several reagents to act as solvent, surfactant and reducing agent, which makes the synthesis process more difficult and expensive. In this paper, we introduced a simple method for the preparation of magnetite nanoparticles using only iron (III) acetylacetonate as metal precursor and oleic acid for all the other functions. This is possible due the constant liberation of the acetylacetone formed in the reaction, which favors the formation of the oleate, the responsible for the conditions required to obtain magnetite nanoparticles smaller than $20 \mathrm{~nm}$. By this method, nanoparticles were obtained with an average size of $17 \mathrm{~nm}$ in a narrow size distribution, uniformity of morphology, high crystallinity and functionalized surface. The organic capping layer allows the preparation of stable colloidal solutions in organic solvents and facilitates the posterior use of nanoparticles in films, ferrofluids and nanocomposite preparation.
\end{abstract}

Keywords Iron oxide - Magnetic oxide · Functionalized surface $\cdot$ Thermal decomposition · Colloidal solution · Oleic acidcoated nanoparticles

\section{Introduction}

The search for simple and effective methods for the synthesis of the materials with desired and modulating properties is constant. Since the beginning of the studies with nanomaterials, conditions of synthesis with lower cost and enhanced properties are sought. The synthesis via colloidal process is widely studied due to the control of size and shape caused by compounds chemically or physically adsorbed in the surface of particles, besides the possibility of surface functionalization [1, 2].

Magnetite nanoparticles have interesting properties, such as low toxicity, eco-friendliness and superparamagnetism [3]. These properties allow their use as ferrofluids
$[4,5]$, in catalysis [6, 7], as pigments [8], in nanocomposite preparation $[9,10]$ and in medical diagnostics $[11]$ and treatment $[12,13]$. Typically, the nanoparticles are synthetized by sol-gel method [14,15], coprecipitation or precipitation [16, 17], electrodeposition [18], hydrothermal route [19], and solvothermal route [20, 21].

In the solvothermal route, the use of an organic solvent is a way to stabilize the nanostructures, because the organic molecules remain bound in crystal surface and cause a steric impediment. In addition, the presence of molecules transfers the solubility to the nanoparticles, resulting in a stable colloidal solution [22]. The use of this synthesis method is interesting for scientific and technological fields, such as in the medical area where the

$\triangle$ Cleocir José Dalmaschio, Cleocir.dalmaschio@ufes.br| ${ }^{1}$ Departamento de Ciências Naturais, Centro Universitário Norte do Espírito Santo, Universidade Federal do Espírito, Santo - Rod. BR 101, km 60, São Mateus, ES CEP: 29932-900, Brazil. ${ }^{2}$ International Iberian Nanotechnology Laboratory, Av. Mestre José Veiga, Braga 4715-330, Portugal. ${ }^{3}$ Instituto de Química, Universidade Estadual de Campinas - Unicamp, Caixa Postal 6154, Campinas, SP CEP: 13083-970, Brazil. ${ }^{4}$ Departamento de Química, Centro de Ciências Exatas e de Tecnologia, Universidade Federal de São Carlos, Rodovia Washington Luiz - km 235, São Carlos, SP CEP 13565-905, Brazil. 
most problems are related with surface coatings [23, 24]. Besides that, most applications of magnetite require particles smaller than $20 \mathrm{~nm}$ in a narrow size distribution for the uniform and improved physical and chemical properties [25].

Many methods are reported for the synthesis of magnetite nanoparticles smaller than $20 \mathrm{~nm}$ by solvothermal route. However, these methods using only a metal precursor and another chemical compound for the functions of solvent, reducing agent and stabilizer to obtain nanoparticles smaller than $20 \mathrm{~nm}$, with uniformity of size and shape and functionalized surface are not reported. Most of the related methods use different agents for each function. Sun and Zeng [25] used phenyl ether, oleic acid, oleylamine and 1-2-hexadecanediol to obtain $4 \mathrm{~nm}$ nanoparticles and for obtaining larger particles ( 12 and $16 \mathrm{~nm}$ ) they used a seed-mediated growth method. Haddad et al. [26] and Pereira et al. [27] used benzyl ether, oleic acid and oleylamine to obtain nanoparticles smaller than $20 \mathrm{~nm}$. Hou et al. [20] used ethylene glycol, hydrazine and different stabilizers to obtain nanoparticles with average sizes between 8 and $11 \mathrm{~nm}$, depending on the surfactant.

Here we described a simple method to produce magnetite nanoparticles with size smaller than $20 \mathrm{~nm}$, in a narrow size distribution, with acid oleic-coated surface by solvothermal method using $\mathrm{Fe}(\mathrm{acac})_{3}$ as metal precursor and oleic acid for all other functions (solvent, reducing agent and surfactant). A large morphological and chemical characterization by X-ray diffraction (XRD), transmission electron microscopy (TEM) and Fourier-transform infrared spectroscopy (FTIR) was made to determine the nanoparticles properties.

\section{Experimental procedure}

\subsection{Synthesis of magnetite nanoparticles}

A solution $0.3 \mathrm{~mol} \mathrm{~L}^{-1}$ of iron (III) acetylacetonate [Fe(acac) $)_{3}$ ] using oleic acid (Synth P.A.) as solvent was prepared in a glass recipient, and transferred to a stainless steel reactor. The system was heated to $300{ }^{\circ} \mathrm{C}$ and hold for $24 \mathrm{~h}$ under magnetic stirred in this temperature. The vapor formed in the reaction was constantly released and the pressure of the system has not exceeded 1.5 bar. After cooling to room temperature, acetone was added in the same volume as reaction suspension and the precipitate formed was centrifuged twice with acetone to remove the excess oleic acid and other byproducts ( $3500 \mathrm{rpm}, 30 \mathrm{~min}$ ). The solid obtained was dispersed in organic solvents, such as chloroform and toluene (Synth P.A.), resulting in a magnetic colloidal solution.

\subsection{Characterization of magnetite nanoparticles}

\subsubsection{X-ray diffraction (XRD)}

The crystallinity and the average size of nanocrystals were evaluated by XRD. It was used a diffractometer Rigaku MiniFlex 600 equipped with copper anode emitter $\mathrm{CuK}_{a}$ radiation $(\lambda=0.154 \mathrm{~nm})$ operated at $40 \mathrm{kV}$ and $15 \mathrm{~mA}$ using a detector D/teX Ultra, using scan speed 3 (deg/min).

\subsubsection{Fourier-transform infrared spectroscopy (FTIR)}

For the evaluation of oleic acid bonded at nanoparticles surfaces, the solvent was evaporated, and the magnetite powder were analyzed in a spectrometer Agilent Technologies Cary 360 with attenuated total reflection accessory (ATR).

\subsubsection{Transmission electron microscopy (TEM)}

The structural, morphological and chemical composition by scanning transmission electron microscopy (STEM) and energy dispersive X-ray spectroscopy (EDX) were evaluated by transmission electron microscopy using a FEI Titan Cubed Themis, with double correction and voltage acceleration of $200 \mathrm{kV}$.

\section{Results and discussion}

The structural information of synthetized nanoparticles indicates that was obtained magnetite in a pure phase, with high crystallinity, according XRD pattern (Fig. 1). The crystallite size was $17 \mathrm{~nm}$, calculated by the Scherrer equation [28].

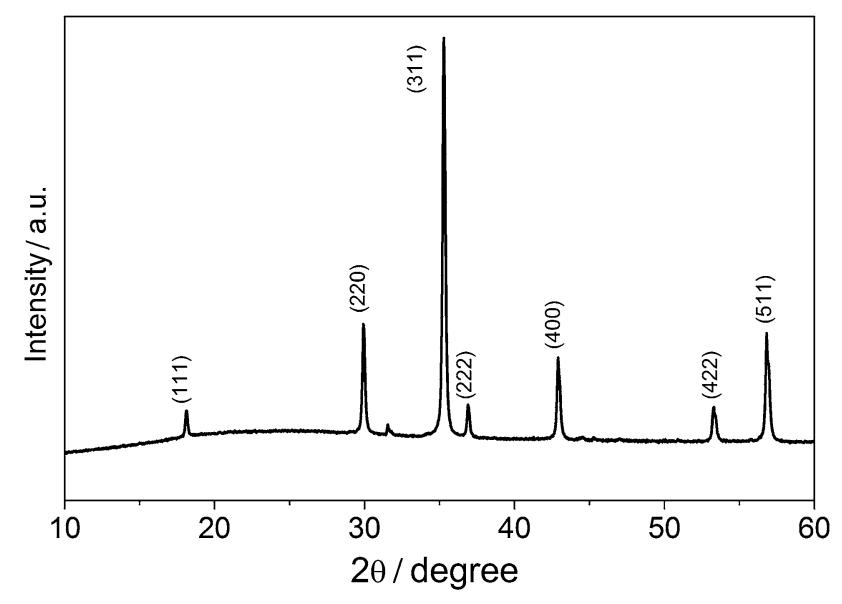

Fig. 1 Diffractogram of iron oxide nanoparticles synthesized indicating the magnetite crystalline phase (the peaks were indexed from PDF \#19-629) 
TEM images (Fig. 2a) shows the uniformity of size and shape of synthesized nanoparticles and the dispersibility. The graph of size distribution (Fig. 2b) shows that the average size is $16.84 \mathrm{~nm}$, the same value estimated from XRD by Scherrer equations, as mentioned. Moreover, from the image and standard deviation it is observed a narrow size distribution. Therefore, the nanoparticles dispersed in solution has potential to the magnetite applications where narrow size distribution are required.

Energy dispersive X-ray spectroscopy in scanning transmission electron microscope (EDX-STEM) was used to analyze sample composition and dispersion of iron and oxygen in the sample. The nanoparticles with high crystallinity have iron and oxygen uniformly distributed, as shows in Fig. 3.
Characterization, structural and morphological, indicates the successful in the synthesis methods using only precursor acetylacetonate and oleic acid. Besides that, literature indicates the use of additional components to control the growth and other components to promotes reduction of iron, then obtain controlled narrow size distribution and well crystalline magnetite nanoparticles. The proposed method in this synthesis protocol can be considered as a simple method to obtain controlled nanoparticles. Details about methods were analyzed considering each component as described in next section.

\subsection{Rule of chemical components}

Uniformity in size and shape of nanoparticles are associated with solvothermal synthesis method and the ligand
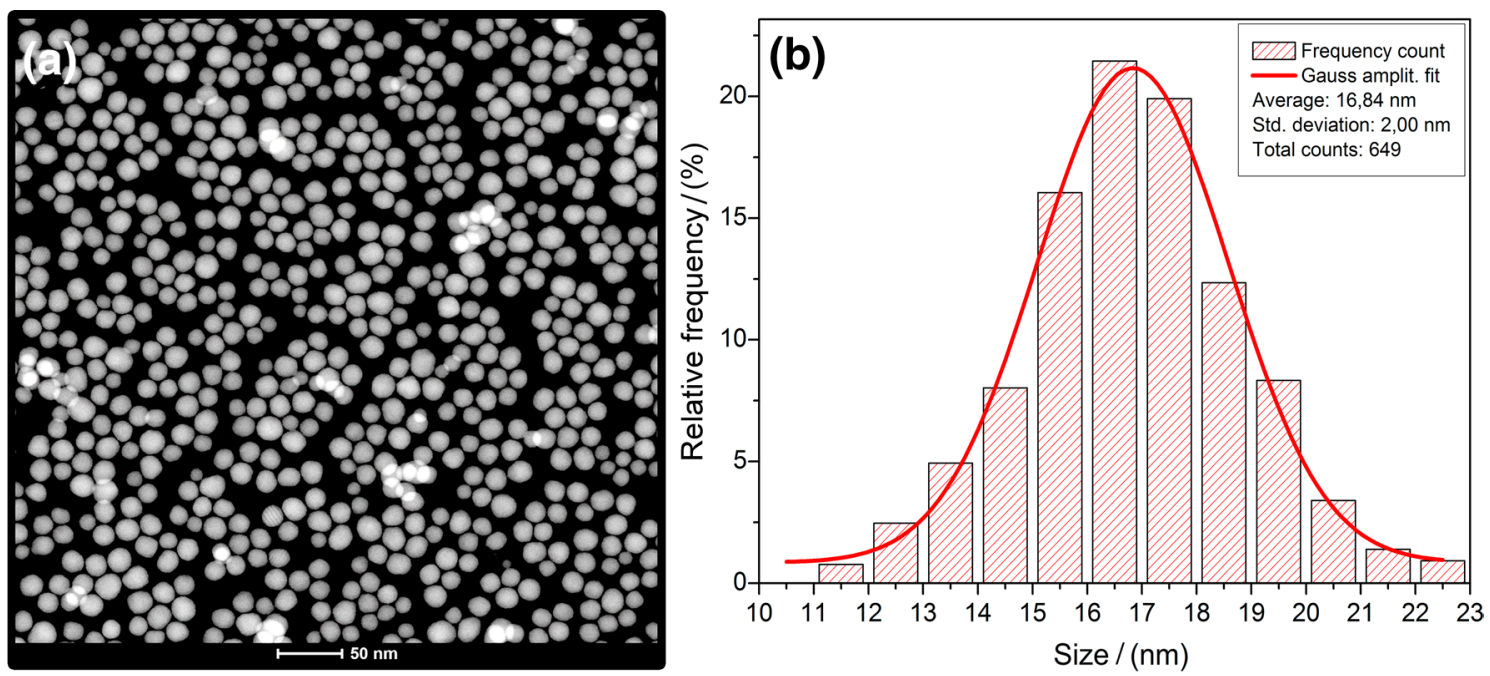

Fig. 2 NCs size distribution from TEM image. a TEM of magnetite nanoparticles synthesized by solvothermal method; $\mathbf{b}$ Graph size distribution obtained from counts in TEM image
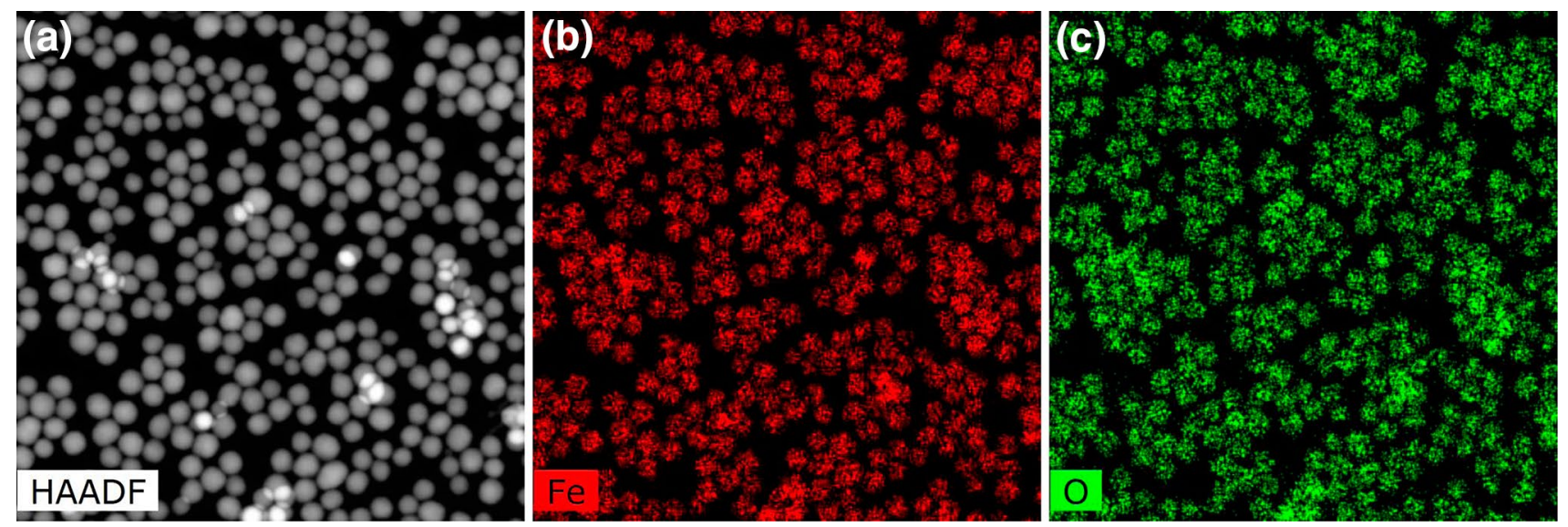

Fig. 3 EDX-STEMimage of magnetite nanocrystals, were the identified elements are indicated in the spectrum. a STEM image of nanocrystals; b Iron map image; c Oxygen map image 
agent used to control the growth. These effects can be considered separated or together [29]. From approach used in this work, during reaction the nanoparticles are functionalized with organic molecules of solvent, that act as ligand over nanoparticle surface, besides the presence of the oleic acid being essential for the process of partial reduction of the $\mathrm{Fe}^{3+}$ ion to $\mathrm{Fe}^{2+}$, to compose particles of magnetite with a correct relation between these ions. So, the conversion of the $\mathrm{Fe}(\mathrm{acac})_{3}$ into $\mathrm{Fe}_{3} \mathrm{O}_{4}$ nanoparticles can be divided in five steps, as will be described in the following sections and schematically presented in Fig. 4 .

\subsection{Formation equilibrium of the reaction intermediate}

\subsubsection{Oleic acid as a solvent}

In the first step, the precursor $\mathrm{Fe}(\mathrm{acac})_{3}$ is solubilized in oleic acid and reacts with it. During this process, an exchange occurs between the acetylacetonate ion and the oleate ion formed by the deprotonation of the oleic acid. The Fe(acac) is converted to iron oleate, soluble in the reaction media, and the acetylacetonate ion is then converted to acetylacetone (Hacac). In fact, the system establishes a chemical equilibrium between oleate and acetylacetonate as described by Eq. (1). In a closed system, under pressure, the equilibrium will be dislocated to left, once that acetylacetonate is a bidentate ligand resulting in six members chelate ring while oleate is a monodentate ligand. So, it is important to release the gas formed in the reaction to promote the formation of the iron oleate in bigger quantity. For this, in reactional conditions, the pressure must be kept lower than 2 bar to eliminate acetylacetone. Besides that, the oleic acid is present in large quantities because it is the solvent, which does not limit the reaction.
Considering the chemical equilibrium and synthesis conditions, it is possible to synthesize shape controlled crystalline magnetite nanoparticles, under adequate conditions. As mentioned in the introduction, the majority synthesis protocol describes a significant complex system using different reducing agents, surfactant and solvents, if compared a synthesis involving only oleic acid and iron acetylacetonate. Also, a quick and indiscriminate release of acetylacetone from reactional system, promotes the rapid conversion of the oleate, resulting in hematite or requires additional chemical components to obtain magnetite. On the other hand, if the reaction was processed in a closed system, with pressures eluted acetylacetone, would lead to the formation of wustite phase (FeO).

\subsubsection{Oleic acid as a reducing agent in the formation of the mixed oxide}

In the second step, occurs the partial reduction of the $\mathrm{Fe}^{3+}$ ions. This step can occur simultaneously with the first step. As mentioned, the presence of a reducing agent is necessary for the $\mathrm{Fe}^{3+}$ reduction to obtain $\mathrm{Fe}^{2+}$, and the oleic acid can be used to this function, as described by Kwon and collaborators [30] and after by Kemp and collaborators [31]. The formation of $\mathrm{Fe}^{2+}$ is initiated at approximately $180^{\circ} \mathrm{C}$ and completed at $320^{\circ} \mathrm{C}$ by two main mechanisms: the oxidative decarboxylation and the 1-octadecene oxidation. The first is based in an oxidative decarboxylation of a metallic carboxylate via homolytic cleavage of the metal-oxygen bond, which forms the reduced metal and the carboxylic radical. This mechanism was evidenced by the detection of by-products of the redox reaction, such as alkanes $\mathrm{C} 8-\mathrm{C} 12$ and alkenes. The other mechanism is the 1-octadecene oxidation and was studied by these groups concluding that this process is also responsible for
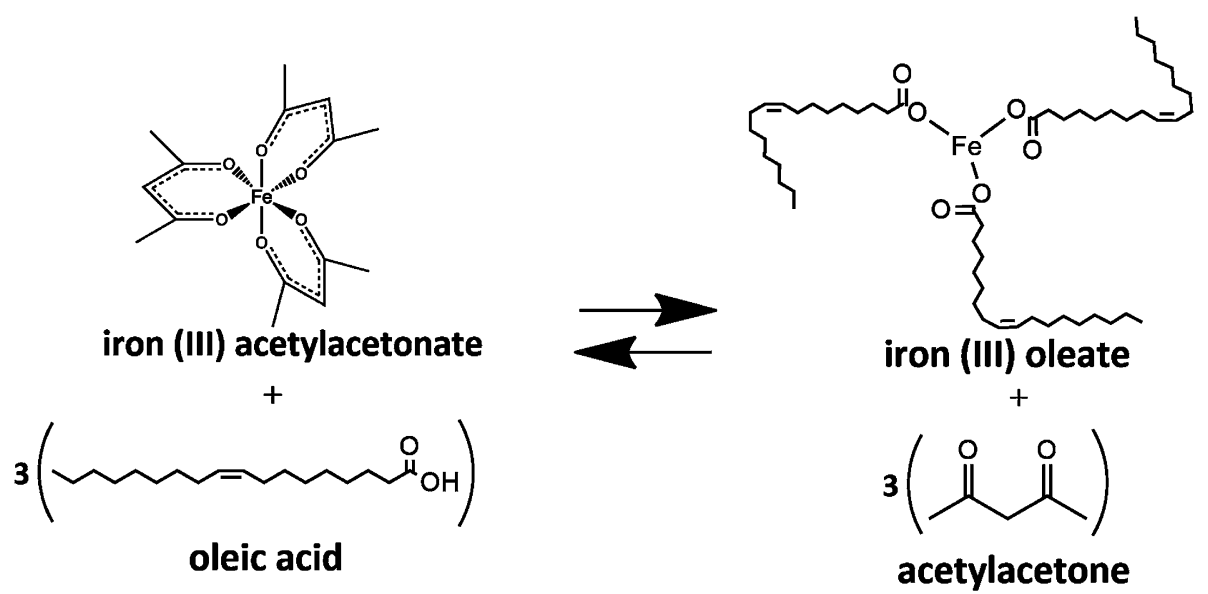
Fig. 4 Scheme representative of mechanism of synthesis and growth of magnetite functionalized nanoparticles

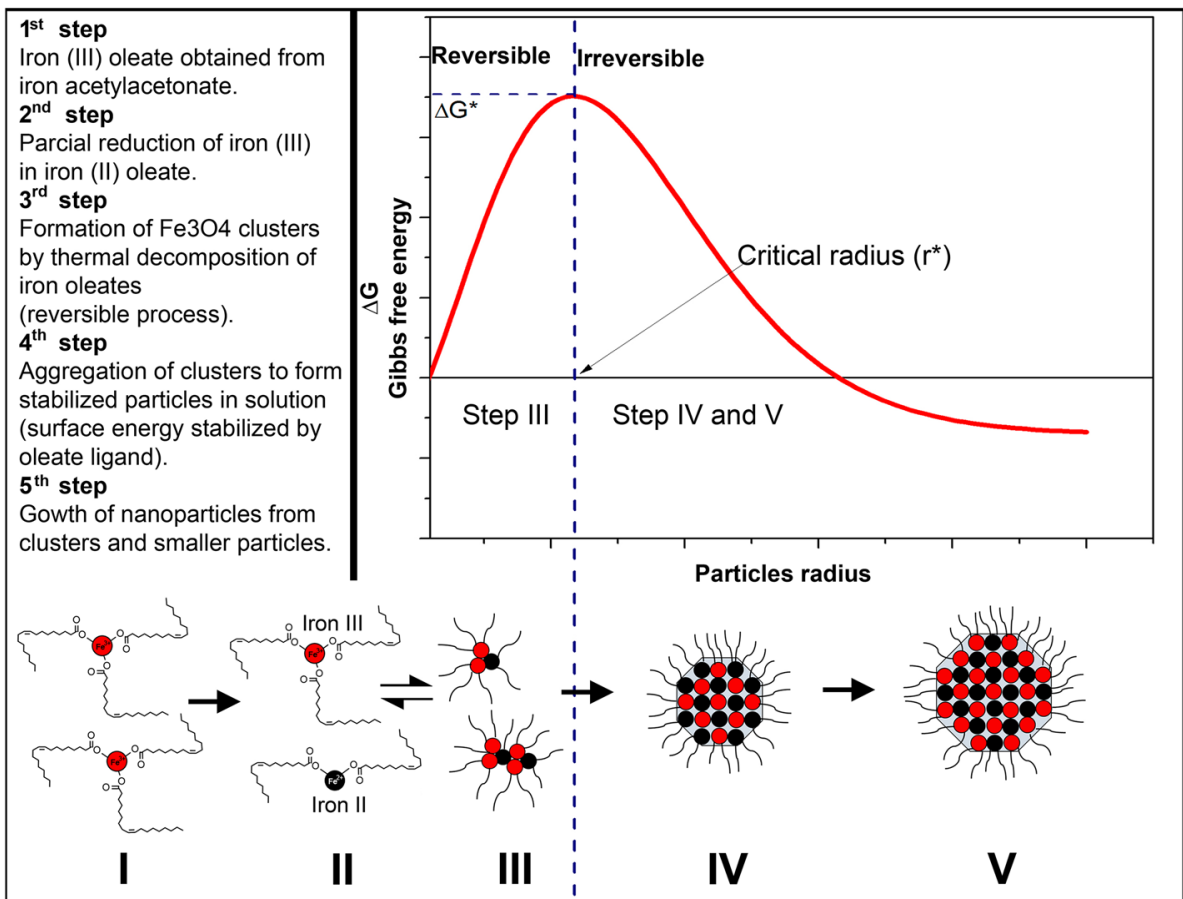

the formation of $\mathrm{Fe}^{2+}$. In this work, as only oleic acid was used with potential reducing agent, the first mechanism of reduction was presented.

\subsubsection{Oleic acid as a surfactant agent}

The third step is the formation of the $\mathrm{Fe}_{3} \mathrm{O}_{4}$ clusters by the thermal decomposition of the iron oleate. The clusters are aggregated until they have enough $\Delta G$, what makes the process irreversible. The clusters that do not reach this level are solubilized again. The formation and the growing of the nanoparticles are the fourth and the

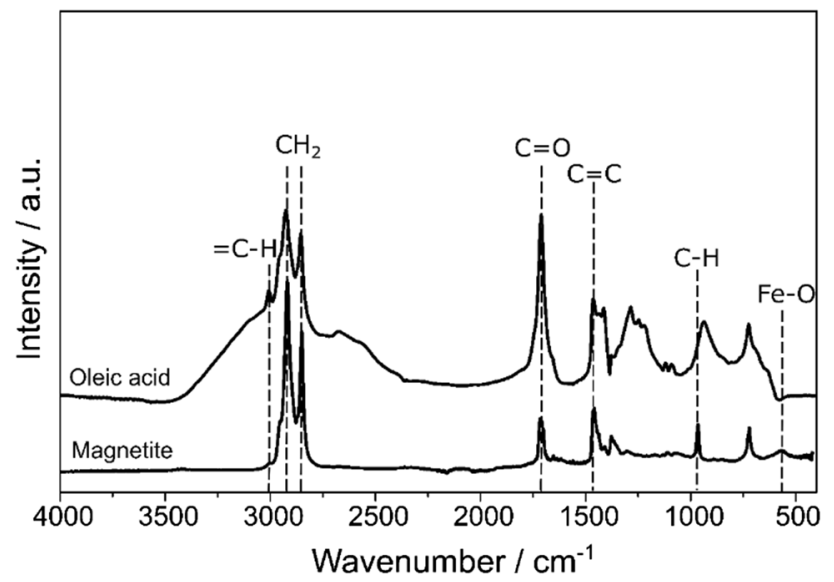

Fig. 5 FTIR spectra from oleic acid and magnetite NP, where similar vibrational modes area observed in predominates peaks in both spectra associated the presence of oleate group acting as ligand over NPs surface fifth steps, respectively. In the fourth step, the oleic acid acts as a surfactant, once that the compound prevents the approximation of the other particles, which would lead to the formation of aggregates. Figure 5 shows the FTIR spectra of the nanoparticles synthetized. As observed, similar vibrational modes are present in oleic acid and in the nanoparticles sample, indicating that the oleate group keeps bounded on the nanoparticle surface after synthesis. Ligands over nanoparticles are bonded from carboxylate group as evidenced: in oleic acid the presence of carboxylic bonds ( $1722 \mathrm{~cm}^{-1}$ region) is intense and it is reduced when compared with spectra from nanoparticle. Furthermore, a dislocation of vibration region in the surface $\left(1710 \mathrm{~cm}^{-1}\right)$ indicating the coordination of carboxylic group on magnetite surface. The vibration of $580 \mathrm{~cm}^{-1}$ region refers to the Fe-O bond in the crystal interior [32]. Therefore, the oleate presence is the factor responsible for stabilization of nanoparticles in their synthesis to obtain uniformity and good dispersibility, as observed in Fig. 2. In addition, the presence of the oleic acid transfers the solubility to the nanoparticles, which results in colloidal solutions after washed and dispersion in organic solvents such as toluene and chloroform.

The action of the oleic acid as surfactant is also evidenced in the analysis of crystallinity and morphology. These details were analyzed by scanning transmission electron microscope (STEM). Figure 6 presents a high magnification of a nanoparticle with typical facets in the crystal surface, as that the energy in facets promotes the crystal shape. The oleate ligand promotes the control in 
Fig. 6 STEM of magnetite nanocrystal. a low magnififacets in the crystal; b High magnification of nanocrystals presenting the atomic columns in crystals cation where lines indicate
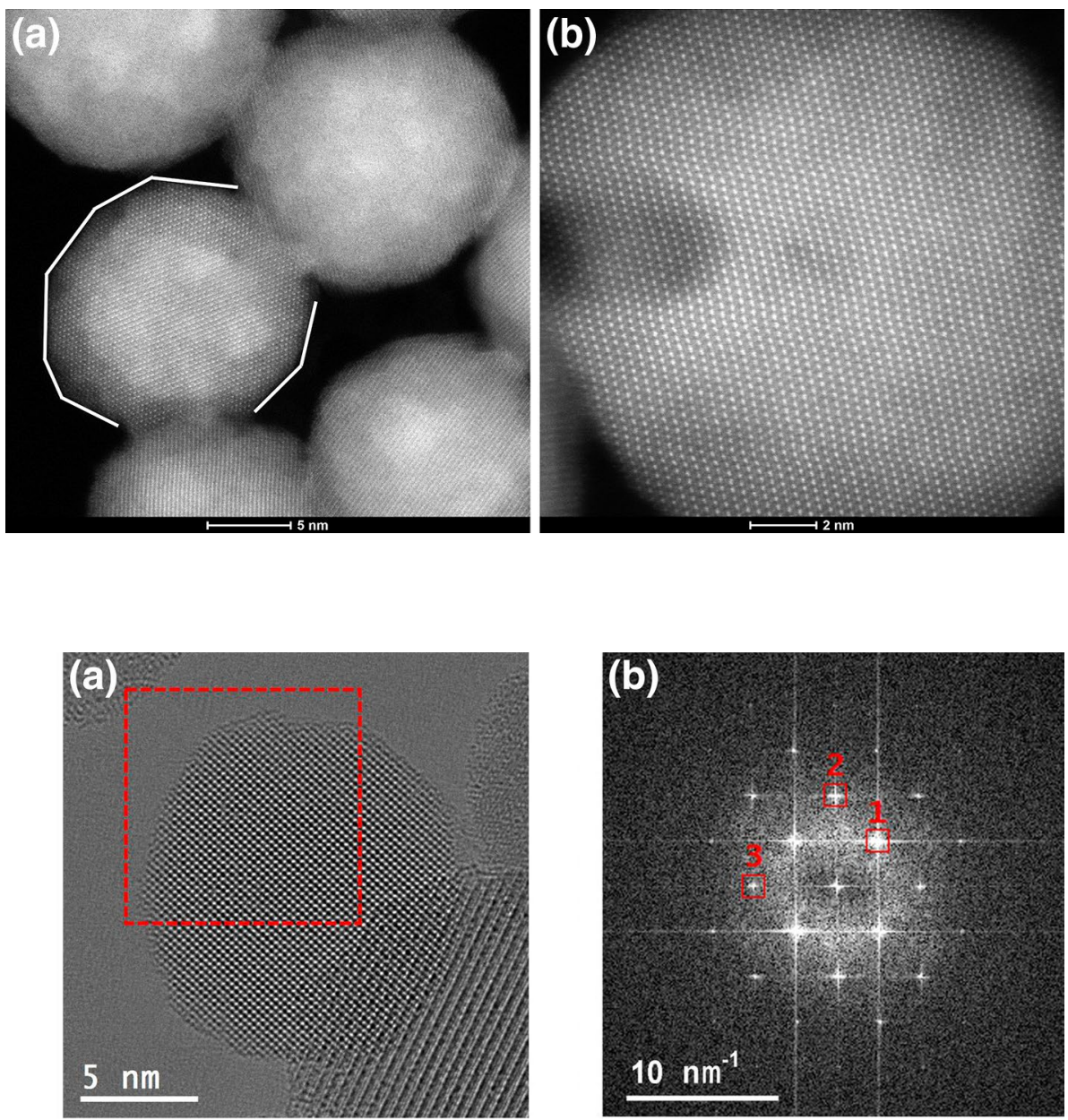

Fig. 7 HRTEM images and simulated images for $\mathrm{Fe}_{3} \mathrm{O}_{4}$. a HRTEM of nanocrystal; $\mathbf{b}$ FFT from image a; c HRTEM and correspondent crystal lattice in zone axis $<5,-1,2>$ from CIF information. Iron-red, Oxygen-yellow; $\mathbf{d}$ unit cell of magnetite in zone axis used to represent atomic positions in c

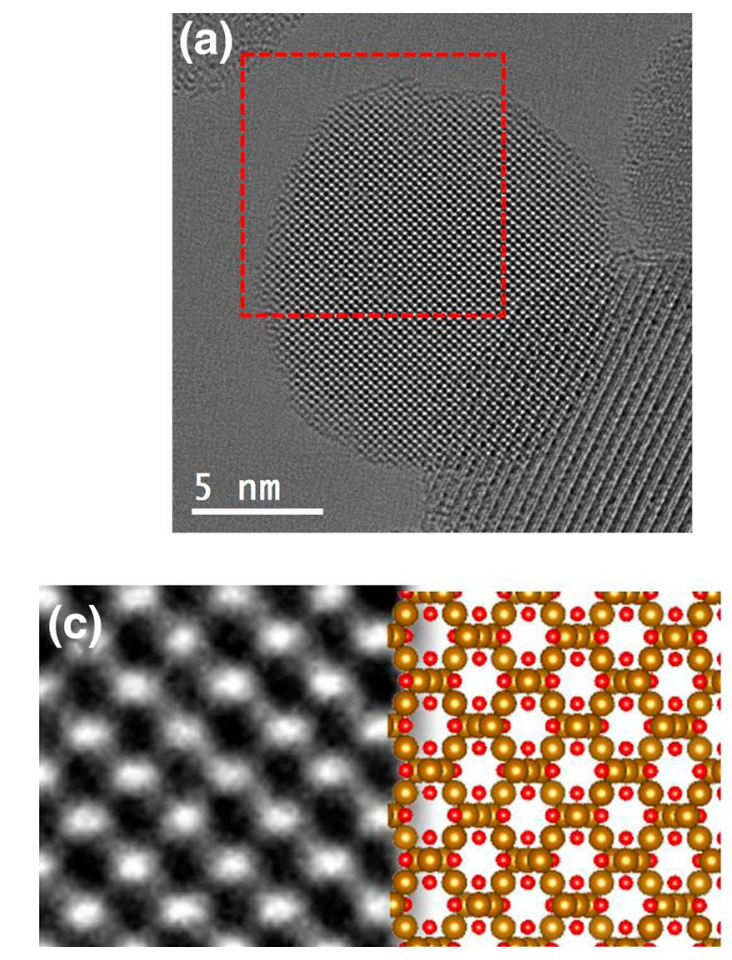

(d)

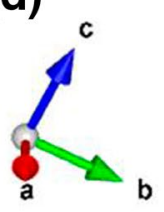

the growth process of the size and shape. Different ligands can be used to control the growth to conduct different shapes. When the same ligand and solvent are present, the ligand desorption and adsorption rate will be low, due the excess of oleic acid, once that it is the solvent. During the reaction, an exchange occurs between the acetylacetonate ion and the oleate ion formed by the deprotonation of the oleic acid. The acetylacetonate ion is then converted to acetylacetone and leaves the system in gaseous form. Thus, the exchange of the acetylacetonate by the oleate is favored and the impediment to the action of the oleate ion is reduced, facilitating its action in stabilizing the shape and size of the magnetite. Therefore, the ligand present on the surface controls the crystal growth in low rate resulting in high crystalline nanocrystals and to oleic acid with facets almost regular to resulting in a faceted tetra dodecahedron [29].

Indexation of crystal lattice were realized from a HRTEM images, as presented in Fig. 7. In the images, a nanocrystal oriented in zone axis $\langle 5,-1,2\rangle$, determined from HRTEM Fast Fourier Transform (FFT) and index using CIF of magnetite, with spots associated with crystalline plane, as indicated in Fig. 7b associated CIF (crystallographic Information File to $\mathrm{Fe}_{3} \mathrm{O}_{4}$ - PDF \#19-629). HRTEM and 
representation of lattice from CIF, are presented in Fig. 7c and $d$, match HRTEM image with atomic positions in represented crystalline lattice in zone axis, as indicated in the Figure.

Hence, the nanoparticles obtained under these conditions have characteristics that allow their application without any additional chemical modification. The hydrophobic nature of the nanoparticle surface allows an improvement in the compatibilization between the nanoparticle and organic matrices. Another possibility to be studied is the exchange of surface ligands by hydrophilic groups, which would facilitate dispersion in aqueous media for biological studies and applications.

\section{Conclusion}

In this work we presented a simple method for synthesis of magnetite nanoparticles with size smaller than $20 \mathrm{~nm}$ using only two reagents: $\mathrm{Fe}(\mathrm{acac})_{3}$ and oleic acid. This was possible due the constant liberation of the acetylacetone formed in the conversion of $\mathrm{Fe}(\mathrm{acac})_{3}$ into iron (III) oleate, which promotes displacement of the equilibrium to obtain greater quantities of the reaction intermediate. Furthermore, iron oleate acts in the reduction of $\mathrm{Fe}^{3+}$ to $\mathrm{Fe}^{2+}$ and in the control of the nucleation and growing of the nanoparticles. The techniques of chemical and morphological characterization showed the uniformity in size and shape of the nanoparticles, high crystallinity, narrow size distribution and good dispersibility in organic solvents, due the presence of organic capping layer in the nanoparticle surface after the synthesis process. These properties obtained by these conditions make possible the use of the nanoparticles in different applications, such as medical area, nanocomposite preparation, film deposition, among others.

Acknowledgements The authors thank the Conselho Nacional de Desenvolvimento Científico e Tecnológico (CNPq), Fundação de Amparo à Pesquisa e Inovação do Espírito Santo (Fapes) and Federal University of Espírito Santo (Ufes) for the research funding and the scholarships.

\section{Compliance with ethical standards}

Conflict of interest The authors declare that they have no competing interests.

\section{References}

1. Jun YW, Choi JS, Cheon J (2006) Shape control of semiconductor and metal oxide nanocrystals through nonhydrolytic colloidal routes. Angew Chem Int Ed 45(21):3414-3439. https://doi. org/10.1002/anie.200503821
2. Yin Y, Alivisatos AP (2005) Colloidal nanocrystals synthesis and the organic-inorganic interface. Nature 437(7057):664-670. https://doi.org/10.1038/nature04165

3. Ai L, Zhang C, Chen Z (2011) Removal of methylene blue from aqueous solution by a solvothermal-synthesized graphene/ magnetite composite. J Hazard Matter 192(3):1515-1524. https ://doi.org/10.1016/j.jhazmat.2011.06.068

4. Bahiraei $M$, Hangi $M$ (2015) Flow and heat transfer characteristics of magnetic nanofluids: a review. J Magn Magn Mater 374:125138. https://doi.org/10.1016/j.jmmm.2014.08.004

5. Hiergeist R, Andrä W, Buske N, Hergt R, Hilger I, Richter U, Kaiser W (1999) Application of magnetite ferrofluids for hyperthermia. J Magn Magn Mater 201:420-422. https://doi.org/10.1016/ S0304-8853(99)00145-6

6. Baeza A, Guillena G, Ramón DJ (2016) Magnetite and metalimpregnated magnetite catalysts in organic synthesis: a very old concept with new promising perspectives. Chem Cat Chem 8(1):49-67. https://doi.org/10.1002/cctc.201500854

7. Gawande MB, Branco PS, Varma RS (2013) Nano-magnetite $\left(\mathrm{Fe}_{3} \mathrm{O}_{4}\right)$ as a support for recyclable catalysts in the development of sustainable methodologies. Chem Soc Rev 42(8):3371-3393. https://doi.org/10.1039/c3cs35480f

8. Froment $\mathrm{F}$, Tournié $\mathrm{A}$, Colomban $\mathrm{P}$ (2007) Raman identification of natural red to yellow pigments: ochre and iron-containing ores. J Raman Spectrosc 38:1538-1553. https://doi.org/10.1002/jrs

9. El-Nahas AM, Salaheldin TA, Zaki T, El-Maghrabi HH, Marie AM, Morsy SM, Allam NK (2017) Functionalized cellulose-magnetite nanocomposite catalysts for efficient biodiesel production. Chem Eng J 322:167-180. https://doi.org/10.1016/j. cej.2017.04.031

10. Sun H, Cao L, Lu L (2011) Magnetite/reduced graphene oxide nanocomposites: one step solvothermal synthesis and use as a novel platform for removal of dye pollutants. Nano Res 4(6):550 562. https://doi.org/10.1007/s12274-011-0111-3

11. Tian Y, Yu B, Li X, Li K (2011) Facile solvothermal synthesis of monodisperse $\mathrm{Fe}_{3} \mathrm{O}_{4}$ nanocrystals with precise size control of one nanometre as potential MRI contrast agents. J Mater Chem 21(8):2478-2481. https://doi.org/10.1039/c0jm02913k

12. Ehlerding EB, Grodzinski P, Cai W, Liu CH (2018) Big potential from small agents: nanoparticles for imaging-based companion diagnostics. ACS Nano 12:2106-2121. https://doi.org/10.1021/ acsnano.7b07252

13. Ho D, Sun $X$, Sun $S$ (2011) Monodisperse magnetic nanoparticles for theranostic applications. Acc Chem Res 44(10):875-882. https://doi.org/10.1021/ar200090c

14. Lu Y, Yin Y, Mayers BT, Xia Y (2002) Modifying the surface properties of superparamagnetic iron oxide nanoparticles through a sol-gel approach. Nano Lett 2(3):183-186. https://doi. org/10.1021/nl015681q

15. Xu J, Yang H, Fu W, Du K, Sui Y, Chen J, Zeng Y, Li M, Zou G (2007) Preparation and magnetic properties of magnetite nanoparticles by sol-gel method. J Magn Magn Mater 309(2):307-311. https://doi.org/10.1016/j.jmmm.2006.07.037

16. Petcharoen K, Sirivat A (2012) Synthesis and characterization of magnetite nanoparticles via the chemical co-precipitation method. Mater Sci Eng C 177(5):421-427. https://doi. org/10.1016/j.mseb.2012.01.003

17. Rajput S, Pittman CU, Mohan D (2016) Magnetic magnetite $\left(\mathrm{Fe}_{3} \mathrm{O}_{4}\right)$ nanoparticles synthesis and applications for lead $\left(\mathrm{Pb}^{2+}\right)$ and chromium $\left(\mathrm{Cr}^{6+}\right)$ removal from water. J Colloid Interface Sci 468:334-346. https://doi.org/10.1016/j.jcis.2015.12.008

18. Wang CY, Zhu GM, Chen ZY, Lin ZG (2002) The preparation of magnetite $\mathrm{Fe}_{3} \mathrm{O}_{4}$ and its morphology control by a novel arcelectrodeposition method. Mater Res Bull 37(15):2525-2529. https://doi.org/10.1021/am4022076 
19. Nadoll P, Angerer T, Mauk JL, French D, Walshe J (2014) The chemistry of hydrothermal magnetite: a review. Ore Geol Rev 61:1-32. https://doi.org/10.1016/j.oregeorev.2013.12.013

20. Hou Y, Yu J, Gao S (2003) Solvothermal reduction synthesis and characterization of superparamagnetic magnetite nanoparticles. J Mater Chem 13(8):1983-1987. https://doi.org/10.1039/ b305526d

21. Kumari M, Pittman CU, Mohan D (2015) Heavy metals [chromium (VI) and lead (II)] removal from water using mesoporous magnetite $\left(\mathrm{Fe}_{3} \mathrm{O}_{4}\right)$ nanospheres. J Colloid Interface Sci 442:120-132. https://doi.org/10.1016/j.jcis.2014.09.012

22. Dalmaschio CJ, Firmiano EGS, Pinheiro AN, Sobrinho DG, de Moura AF, Leite ER (2013) Nanocrystals self-assembled in superlattices directed by the solvent-organic capping interaction. Nanoscale 5:5602-5610. https://doi.org/10.1016/10.1039/ c3nr00883e

23. Ali A, Zafar H, Zia M, ul Haq I, Phull AR, Ali JS, Hussain A (2016) Synthesis, characterization, applications, and challenges of iron oxide nanoparticles. Nanotechnol Sci Appl 9:49-67. https://doi. org/10.2147/NSA.S99986

24. Li J, Shige W, Shi X, Shen M (2017) Aqueous-phase synthesis of iron oxide nanoparticles and composites for cancer diagnosis and therapy. Adv Colloid Interface Sci 249:374-385. https://doi. org/10.1016/j.cis.2017.02.009

25. Sun S, Zeng H (2002) Size-controlled synthesis of magnetite nanoparticles. J Am Chem Soc 124(28):8204-8205. https://doi. org/10.1021/ja026501x

26. Haddad PS, Martins TM, D'Souza-Li L, Li LM, Metze K, Adam RL, Knobel M, Zanchet D (2008) Structural and morphological investigation of magnetic nanoparticles based on iron oxides for biomedical applications. Mater Sci Eng, C 28(4):489-494. https ://doi.org/10.1016/j.msec.2007.04.014

27. Pereira GFL, Costa FN, Souza JA, Haddad OS, Ferreira FF (2018) Parametric Rietveld refinement and magnetic characterization of superparamegnetic iron oxide nanoparticles. J Magn Magn Mater 456:108-117. https://doi.org/10.1016/j. jmmm.2018.02.020

28. Cullity BD (1978) Elements of X-ray diffraction. Addison-Wesley Publishing Company, Michigan

29. Qiao L, Fu Z, Li J, Ghosen J, Zeng M, Stebbins J, Prasad PN, Swihart MT (2017) Standardizing size- and shape-controlled synthesis of monodisperse magnetite $\left(\mathrm{Fe}_{3} \mathrm{O}_{4}\right)$ nanocrystals by identifying and exploiting effects of organic impurities. ACS Nano 11(6):6370-6381. https://doi.org/10.1021/acsnano.7b027 52

30. Kwon SG, Piao Y, Park J, Angappane S, Jo Y, Hwang NG, Park JG, Hyeon T (2007) Kinects of monodisperse iron oxide nanocrystal formation by "heating-up" process. J Am Chem Soc 129:1257112584. https://doi.org/10.1021/ja074633q

31. Kemp SJ, Ferguson RM, Khandhar AP, Krishnan KM (2016) Monodisperse magnetite nanoparticles with nearly ideal saturation magnetization. RSC Adv 6:77452-77464. https://doi. org/10.1039/c6ra12072e

32. Atta AM, El-Mahdy GA, Al-Lohedan HA, Al-Hussain S (2014) Synthesis of environmentally frindly highly dispersed magnetite nanoparticles based on rosin cationic surfactants as thin film coatings of steel. Int J Mol Sci 15:6974-6989. https://doi. org/10.3390/ijms15046974 